\title{
PAPER
}

\section{Infants' use of social partnerships to predict behavior}

\section{Marjorie Rhodes, ${ }^{1}$ Chelsea Hetherington, ${ }^{2}$ Kimberly Brink ${ }^{3}$ and Henry M. Wellman ${ }^{3}$}

1. Department of Psychology, New York University, USA

2. Institute of Child Development, University of Minnesota, USA

3. Department of Psychology, University of Michigan, USA

\begin{abstract}
The experiences of social partners are important motivators of social action. Can infants use such experiences to make predictions about how social agents will behave? Sixteen-month-old infants were introduced to two social pairs. Initial events established within-pair cooperation as well as between-pair conflict involving an individual from each pair. Following these events, infants looked longer when between-pair members who had never previously interacted now cooperated - instead of conflicted - with each other. Thus, infants tracked the third-person allegiances and inferred that the conflict would generalize across social partnerships. These findings demonstrate a critical feature of early social cognition and promote needed, further research on the role of social allegiances in social cognition across development.
\end{abstract}

\section{Introduction}

Human action is often motivated by the experiences of social partners. Imagine that two children - Jane and Molly - have an argument. Another child, Jill, sees the conflict, and later initiates an argument with Molly. This incident can be understood - and even predicted - if we (1) know that Jill is friends with Jane (or otherwise has a cooperative allegiance with her, e.g. perhaps they have previously helped one another, or are on the same sports team), (2) expect social partners to be influenced by each other's experiences, and thus (3) infer that given Jane's conflict with Molly, Jill will be unfriendly towards Molly too. Tracking social allegiances and monitoring the experiences of social partners are crucial strategies for explaining and predicting behavior. Indeed, behavioral patterns consistent with this example - in which social partners act on each other's behalf - are plentiful in experimental social psychology research (Batson, 1998), and in observational (e.g. of patterns of schoolyard bullying; Hodges, Boivin, Vitaro \& Bukowski, 1999; of aggression in non-human primates; Cheney \& Seyfarth, 1986, 1999) and sociological studies (e.g. the spreading of violence across urban gangs; Papachristos, 2009). Adult psychologists as well as laypersons (Batson, 1998) regularly form expectations about others' behavior by monitoring social allegiances.

Do human infants use the experiences of social partners to form expectations about how agents will behave? That is, do infants track social allegiances? This is a surprisingly neglected question. By the second year of life, infants appeal to a range of unobservable causal factors when forming expectations about human action, including goals (Woodward, 1998), desires (Repacholi \& Gopnik, 1997) and beliefs (Onishi \& Baillargeon, 2005). By focusing on the role of individual mental states, however, this research has not examined whether infants use broader aspects of social structure (e.g. social partnerships, group memberships) to understand and predict behavior.

This represents an important theoretical gap. Understanding whether infants incorporate social allegiances into their expectations about behavior is necessary to establish both the origins of social cognition and the nature of developmental change. One possibility is that recognition of the inferential value of social partnerships 
stems from children's own experiences with social partners (similar to the example above). By the preschool years, children use social partnerships to predict certain emotional states (Pietraszewski \& German, 2013) and behaviors (Chalik \& Rhodes, 2014). For example, 4-yearolds expect people to become angry with those who harm their friends (Pietraszewski \& German, 2013) and expect group members to retaliate on each other's behalf following conflict among individuals from different groups (Chalik \& Rhodes, 2014). Because infants in the second year of life have yet to form their own stable friendships or cooperative partnerships outside of the family (Platten, Hernik, Fonagy \& Fearon, 2010), however, understanding coalitions and alliances could be a later developing component of social cognition, developing only post-infancy. Alternatively, monitoring the experiences of social partners and using such experiences to predict behavior could contribute to, as well as result from, early childhood social interactions, and therefore be evident even in infancy. If so, this would reveal an important continuity in social cognition across development.

A few studies indicate that infants have some systematic expectations about patterns of social interaction. For example, securely attached 12-month-old infants expect maternal-like agents to approach and provide help to rather than abandon - baby-like agents (Johnson, Dweck \& Chen, 2007). Ten-month-olds expect individuals to approach agents who have helped them in the past over agents who have harmed them (Hamlin, Wynn \& Bloom, 2007; Kuhlmeier, Wynn \& Bloom, 2003), and 9-montholds expect people who have similar food preferences to interact positively but those with dissimilar preferences to interact negatively (Liberman, Kinzler \& Woodward, 2014). By 18 months, toddlers expect someone to provide help to a friend who is in distress (Beier, Carpenter $\&$ Tomasello, 2011). Yet, none of these previous studies have tested whether infants make inferences about the behavior of agents based on the experiences of the agents' social partners. Here, instead of asking whether infants expect individuals to come into conflict with those whom they have fought with in the past (along the lines of prior work), we test whether infants expect individuals to come into conflict with those whom their social partners have fought with in the past. The present research thus examines broader understandings and potentially more powerful social expectations than have been documented in prior infant work. The present studies test whether infants use the experiences of social partners to form expectations about how third parties will behave, and specifically whether infants expect conflict (rather than cooperation) between two agents based on the interactions of other related parties.

\section{Study 1a}

\section{Method}

Participants included 33 infants $^{1}$ (17 male; $M$ age $=$ 15.92 months, range $=15.53-16.47$ months) randomly assigned to the Conflict condition $(N=16)$ or the Cooperation condition $(N=17)$. Eight additional infants were excluded for fussiness $(n=4)$, parent interference $(n=2)$, computer problems $(n=1)$, and low interest in the initial events $(n=1)$.

The stimuli were a series of videoed events. Infants' looking times were coded by a live observer. A secondary observer coded $33 \%$ of participants. Observer agreement was within 1 second or less on $88.3 \%$ of trials in Study 1a ( $92 \%$ for Study $1 \mathrm{~b} ; 85 \%$ for Study $1 \mathrm{c} ; 90 \%$ for Study $2 \mathrm{a}$; $83 \%$ for Study $2 b ; 93 \%$ for Study 3a; $82 \%$ for Study $3 b$ ). The setting included a cloth-covered table, with a transparent box in the middle containing a clearly visible multicolored ball (see Figure 1). The events involved four characters: an orange cat (Cat-A), a grey cat (CatB), a multicolored dog (Dog-A), and a black dog (DogB). The pair of cats wore red bandanas and the pair of dogs wore white bandanas. The events were shown in three phases in a violation-of-expectation paradigm. The first two phases introduced and established the partnerships by displaying several interactions, and the last

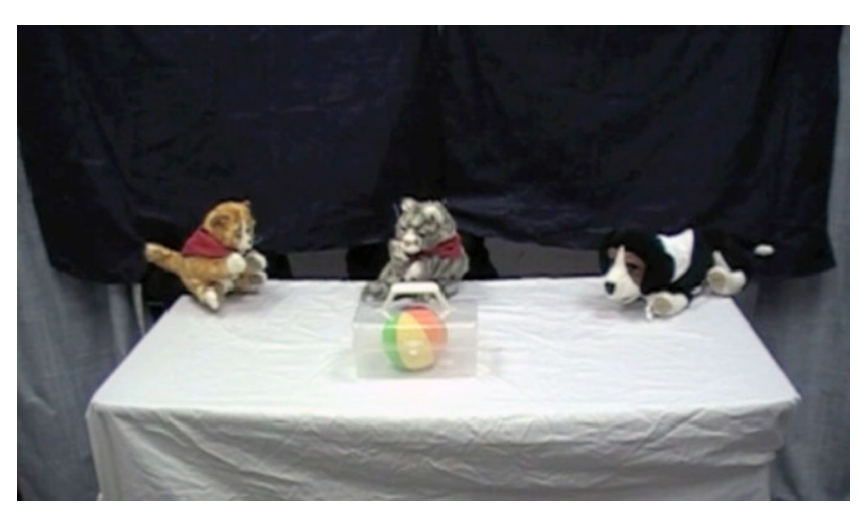

Figure 1 Photograph of display, Study 1.

\footnotetext{
${ }^{1}$ Our target sample size for all studies was $n=24$. Because we expect that some infants who are scheduled to participate will not provide usable data (e.g. because they miss their appointment or do not complete the experiment), we schedule more participants in advance to ensure an adequate sample size. Sometimes, this leads to a larger sample size than intended, as well as to variability in the sample size across studies. Here, we report all collected data; however, analyses on all data sets using only the first 24 participants yield identical patterns of significant results.
} 
phase tested expected interactions about two agents who had never previously interacted but whose partners had.

\section{Phase 1: Introduction}

Phase 1 involved three introductory events: (1) the pair of dogs entered from the back of the stage, approached the box, looked at it with interest and then left through the back, (2) the pair of cats entered from the back of the stage, approached the box, looked at it with interest and then left through the back, and (3) Dog-A entered through the back of the stage and sat in the back right corner of the table, Cat-A entered through the back of the stage and sat in the back left corner of the table (see Figure 1).

\section{Phase 2: Establishing relationships}

The second phase presented the relevant social partnerships and interactions. To help infants track the social partnerships, multiple cues were provided: species (one partnership included two dogs, the other two cats), a visual feature (one partnership wore white bandanas, the other wore red) and social behaviors (the members of each partnership helped each other obtain a goal). Crucially, the events were designed to show that the two dogs were cooperative social partners and the two cats were cooperative social partners. The events also presented an instance of conflict between one member of each partnership. The events used to establish cooperation and conflict built on methods used by Hamlin and Wynn (2011).

As schematized in Figure 2, at the beginning of each event, Dog-A sat in the back right corner of the stage and Cat-A sat in the back left corner of the stage. Then, either Dog-B or Cat-B (referred to as the target agent) entered from the back of the stage, approached the box, and attempted - but failed - to open it three times. Next, one of the two animals already sitting on the table approached the box, and a series of four events occurred,

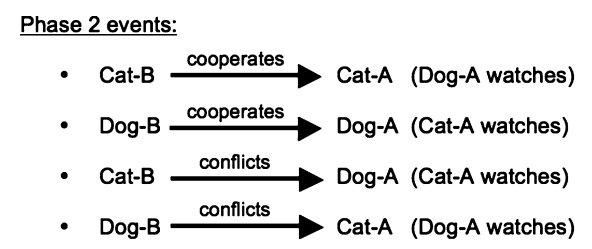

Phase 3 events:
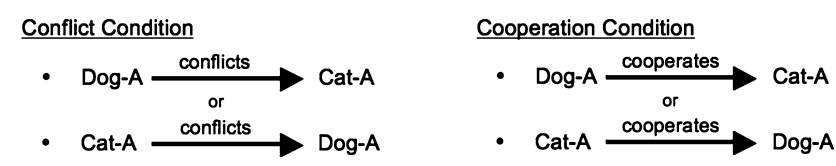

Figure 2 Schematic of events, Study 1. two cooperation events and two conflict events. In a cooperation event, the approaching animal helped the target agent to open the box, took out the toy, and those two animals played with it cooperatively by passing the ball back and forth while the ball made a jingling noise. In a conflict event, the approaching animal prevented the agent from getting the toy by slamming the box shut, then took the ball out himself, and that animal plus the target agent fought over it by pulling it back and forth. Growling sounds (synchronized to the jingling sounds in a cooperation event) were made as the animals pulled the ball back and forth. The pulling (in conflict events) and the passing back and forth (in cooperation events) were also synchronized so that in both displays the animals moved in tandem in a similar manner back and forth on the screen.

There were four events presented in the set order shown in Figure 2. Thus, the first two events established the cooperative pairings, and the second two events established incidents of conflict between one member from each pair. Note that, although Cat-A and Dog-A were present on the stage throughout Phase 2, they never interacted with each other. Critically, all four animals engaged in two action sequences each, once in a cooperative sequence and once in a conflict sequence.

\section{Phase 3: Test events}

Test events began as did Phase 2 events - Cat-A and Dog-A sat alone in the back corners on the stage. This time, however, one of them acted as the target agent, by approaching the box (from the corner), and attempting (but failing) to open it three times. As schematized at the bottom of Figure 2, the other animal on the stage then approached, and initiated either the cooperation or the conflict actions (the same actions as in Phase 2). Each infant saw one type of test event (conflict or cooperation) two times. Test trials ended when infants looked away for 2 continuous seconds or after 60 seconds. Within each condition, approximately half of the infants saw Dog-A as the target agent and half saw Cat-A as the target agent. Thus, the test events differed from Phase 2 in that the target agent was Dog-A or Cat-A (instead of Dog-B or Cat-B) and Dog-A and Cat-A, who had never interacted previously, now interacted.

\section{Results and discussion}

Phase 2 events were presented for a maximum of 33 seconds each. Infants looked equally long at cooperation events (events 1 and 2, $M=30.37, S D=2.58$ ) and conflict events (events 3 and $4, M=29.28, S D=3.69$ ), $t$ 
$(32)=1.67, n s$. Thus, infants were highly interested in both types of events and did not receive more exposure to one type or the other.

If infants have tracked the relevant social partnerships, expect the experiences of an agent's social partners to influence the agent's behavior, and so expect members from different pairs to conflict with each other following conflict between their partners, then infants who see an instance of cross-pair cooperation should look longer at the scene (indicating increased interest and violation of expectation) than infants who see an instance of crosspair conflict. Indeed, infants looked significantly longer in the Cooperation condition $(M=54.99, S D=7.06)$ than in the Conflict condition $(M=44.70, S D=11.81), t$ $(31)=3.06, p=.005, d=1.06$, see Figure 3. Thus, even though infants had never before seen the agents involved in the test trials interact with each other, they found an instance of cross-pair cooperation attention-worthy (unexpected), following an instance of conflict involving those agents' social partners.

\section{Study $\mathbf{1 b}$}

We propose that infants looked longer at the cooperation than conflict test events in Study 1a because of their preceding exposure to the cooperative partnerships. However, perhaps infants just have telling expectations about these specific test events regardless of any prior experimental information. For example, maybe infants, even at this young age, expect that dogs and cats are more likely to fight than cooperate, or that any two animals, generally, are more likely to fight than cooperate. To address this possibility, we examined whether infants look longer at our cooperation than conflict test events in the absence of previous exposure to Phase 2 events.

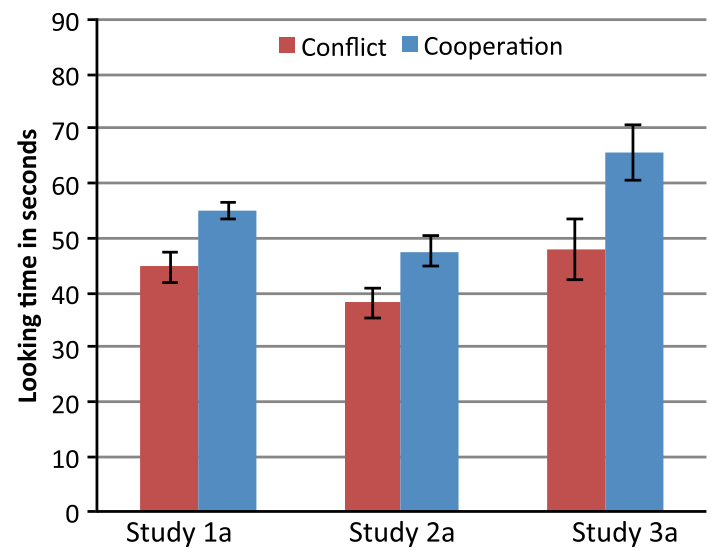

Figure 3 Looking time in seconds to test events, Studies 1a, $2 a, 3 a$. Error bars represent one standard error of the means.
In Study 1b, a new sample of 24 infants (13 male; $M$ age $=15.85$ months, range $=15.53-16.40$ ) were shown the identical test events used in Study 1a, but without any previous exposure to Phase 2 events. Again half saw the conflict test events and half the cooperation (lower part of Figure 2). Unlike in Study 1a, looking time to the test events did not vary by condition (Conflict, $M=42.60, S D=11.98$, Cooperation, $M=46.12, S D=$ 14.67), $t(22)=.83, n s$. Thus, infants did not look longer at the cooperation than conflict test events of Study 1a because they generally found cooperation more unexpected than competition, or cooperation between dogs and cats more interesting or unexpected than conflict between dogs and cats.

\section{Study 1c}

Studies $1 \mathrm{a}$ and $\mathrm{b}$ leave open the question of whether infants are responding to relations between types of animals (e.g. dogs vs. cats) as opposed to actions between individuated agents who also happen to fall into different social partnerships. We address this issue further in Studies 2 and 3 , but in advance and at a minimum we needed to establish whether infants discriminate between the different individual animals. To obtain this evidence, a new group of 26 infants first saw one pair of animals (i.e. the two cats or two dogs) complete the events shown in Phase 1 of Study 1a (i.e. the pair of animals entered, walked up to the box, and looked at it). Subsequently, they saw scenes where initially only the box was on the stage and one individual (e.g. Cat-A) entered the stage from the back, approached the box and tried to open it three times. Then, the scene froze. Infants saw this scene five times all with the same animal (e.g. Cat-A), and then saw the exact same sequence performed by another animal. For half the infants $(N=13,5$ male; $M$ age $=15.74$ months, range $=$ 15.50-16.40) these events involved same-species animals (either two cats, $n=6$; or two dogs, $n=7$ ). For half the infants $(N=13,6$ male; $M$ age $=15.84$, range $=15.53$ 16.40) these events involved a cat and a dog.

As expected, infants' looking time significantly increased when the identity of the agent changed, both when identities changed within a species (e.g. Cat-A to Cat-B; $t(12)=3.23, p=.007, d=.97$, last trial before character change, $M=9.88, S D=6.65$; first trial of new character, $M=17.10, S D=8.11 ; 11$ of 13 infants increased their looking across the key trials, $p=.022$, binomial test) and following the change in species $(t(13)$ $=3.22, p=.007, d=1.25$, last trial before character change, $M=9.20, S D=6.93$; first trial of new character, $M=22.34, S D=14.44 ; 11$ of 13 infants increased their looking across the key trials, $p=.022$, binomial test). 
These data show that infants of this age are capable of discriminating between these individual animals; in Studies 2 and 3 we provide further evidence that they did so in the context of the more complicated Phase 2 and Phase 3 events of our main experiments.

\section{Study 2}

Study 1a presented several cues to the relevant social partnerships: species (dogs vs. cats), social behavior (within-pair cooperation), and perceptual cues (bandana color). Study $1 \mathrm{~b}$ confirmed that infants do not generally expect that animals of different species, or animals marked by different bandana colors, will come into conflict instead of cooperate, but rather formed these expectations because of the previous instances of within-pair cooperation and cross-pair conflict. What types of cues are necessary for infants to track social partnerships in this manner? Of particular importance is whether social interactions alone can define the partnerships for infants or whether infants track social partnerships only when they are consistent with previous category knowledge (e.g. that dogs differ from cats). Study $2 \mathrm{a}$ addresses these possibilities.

\section{Study $2 a$}

Study 2a was identical to Study 1a, with the exception that all of the agents were dogs. To help children track the individual identities and partnerships, each dog had unique coloring and features and one pair of dogs wore red bandanas and the other wore white.

\section{Methods}

Participants were 30 infants $(11$ male; $M$ age $=$ 15.92 months, range $=15.50-16.37) ;$ an additional 2 infants were excluded for fussiness $(n=1)$ or low interest in the initial events $(n=1)$. Procedures were identical to Study 1, except that infants first completed a warm-up to help them individuate the four different dogs. Infants were shown all four puppets and were allowed to hold each one, one at a time. To introduce each dog, the experimenter said, for example, 'Look at this doggy. It's got a red scarf.' In this warm-up, the dogs never interacted. After this, infants were shown the same Phase 1, Phase 2, and test events described in Study 1.

\section{Results}

Infants looked equally long at the cooperation Phase 2 events $(M=29.88, S D=2.60)$ as the conflict Phase 2 events $(M=29.57, S D=2.51), t(29)=.60$, ns. Thus, infants received extensive and equal exposure to both types of events.

Replicating Study 1a, infants looked longer at the test events in the Cooperation condition $(M=47.65, S D=$ 11.11) than in the Conflict condition $(M=38.15, S D=$ $11.32), t(28)=2.32, p=.03, d=.85$; see Figure 3 . Thus, infants tracked social partnerships even when they did not reflect previous category knowledge. Besides this substantive finding, infants' discriminative responding to the test events of Study 2a indicates that they must be differentiating the individual dogs, because otherwise all the agents in Study 2a would be interchangeable.

\section{Study $2 b$}

Study $2 \mathrm{~b}$ addressed whether infants expected the members of the different pairs to come into conflict instead of cooperate because of the Phase 2 events that established the cooperative allegiances, rather than general expectations about how these individual dogs or dogs wearing different colors will interact. Participants were 24 infants (13 Male, $M$ age $=15.9$ months, range $=15.53-16.37)$. Following the same warm-up used in Study 2a, children were shown either the conflict or cooperation test events, with no prior Phase 2 events, as in Study 1b. As expected, infants looked equivalently long at the conflict $(M=32.43, S D=11.32)$ and cooperation $(M=31.11$, $S D=11.56$ ) test events, $t(22)=0.28, n s$. Thus, infants did not look longer in the cooperation than conflict test conditions of Study 2a because they generally found cooperation more interesting or unexpected.

These data also confirm that infants in Study 2a did not form expectations about cross-pair cooperation or conflict simply because of the perceptual differences between the groups (i.e. the different bandana colors), but rather because of the social interactions presented in the Phase 2 events. Thus, although the perceptual feature that marked the pairs (bandana color) may (or may not) have been helpful for allowing infants to track the relevant social partnerships in Study 2a, this perceptual feature alone was not sufficient to set up the key partnership expectations documented there. In Study 2b, bandana color did not lead infants to expect cooperation or conflict; infants formed those expectations only in Study 2a where bandana color was accompanied by information about social interactions.

Together, Studies 1a and 2a confirm that infants form expectations about social behavior based on information about within-pair and cross-pair interactions. Even when the agents were individual members of the same species (rather than from distinctively different species), infants 
monitored partnerships based on the agents' social interactions.

The data across both studies also rule out further alternative interpretations. For example, suppose infants behaved according to a rule such as 'having seen conflict between dogs and cats, expect conflict for other dogs and cats' in Study 1a. Such an approach could not yield the Study 2a findings, because infants had seen both conflict and cooperation among dogs. In general, Studies $1 \mathrm{~b}$ and $2 \mathrm{~b}$ confirm that infants are not merely relying on general expectations about categories (dogs vs. cats) or perceptual markers (bandana color).

\section{Study 3}

In Study 2a, infants tracked social allegiances when partnerships were marked by both bandana color and cooperative interactions. Study $2 \mathrm{~b}$ ruled out the possibility that infants used bandana color alone to form expectations about how members of different pairs would interact with one another. Nevertheless, an important issue remains: perhaps after viewing the Phase 2 events, infants simply inferred that animals with different colored bandanas would conflict with one another. In other words, perhaps they focused so much on the bandana colors that they simply encoded 'one red-wearing dog and one white-wearing dog conflicted' and thus expected a red-wearing dog and white-wearing dog to conflict again, without noticing that the individual identities of the involved parties changed from the Phase 2 to Phase 3 events. To address this issue, we conducted Studies $3 \mathrm{a}$ and $3 \mathrm{~b}$.

\section{Study 3a}

Methods

Participants were 24 infants $(10$ male; $M$ age $=$ 15.86 months, range $=15.50-16.33$ ); an additional four infants were tested but excluded (1 for fussiness, 3 for low interest in the Phase 2 events). Procedures were identical to Study 2a, with three exceptions. First, during the initial warm-up, in which children were allowed to hold each dog (see Study 2a), the dogs began wearing bandanas but the experimenter then removed the bandanas, giving the infant exposure to the dogs without them. As in the previous studies, each dog had individual markings that we expected infants would be able to use to individuate the dogs and track the cooperative partnerships. Second, although the dogs wore bandanas in Phase 1 and Phase 2 (to help infants track the crucial interactions) the dogs wore no bandanas for the critical
Phase 3 test events. Thus, if infants merely formed the expectation that animals with different bandanas would conflict, or failed to encode the individual identities of the agents, then during the test trials they should have no systematic expectations about how particular agents will behave. Third, pilot testing revealed that many infants looked longer at the test events in this study than in the previous two studies, perhaps because it took them longer to identify the relevant social partnerships during the test trials in the absence of a perceptual aid. Therefore, we increased the maximum looking time allowed during the test events from 60 seconds to 90 seconds.

\section{Results}

As in previous studies, infants looked equally at the Cooperation $(M=31.52, S D=1.51)$ and Conflict $(M=30.65, S D=1.93)$ events during Phase 2, $t(23)=$ 1.89 , ns. Replicating Studies 1a and 2a, infants looked longer at the test events in the Cooperation condition $(M=65.73, S D=18.78)$ than in the Conflict condition $(M=47.93, S D=18.31), t(22)=2.34, p=.03$, Cohen's $d=.96$; see Figure 3. Thus, infants tracked social partnerships (perhaps initially aided by bandanas) and applied that partnership information even in the absence of bandana color. In this procedure, infants formed systematic expectations about how the members of the different pairs would interact with each other, even when the individual members of the pairs were no longer marked by bandana colors. Thus, these data further confirm that infants tracked the individual dogs' identities, otherwise they would not have been able to track the social partnerships during the Phase 3 events.

\section{Study $3 b$}

Study $3 \mathrm{~b}$ was designed to confirm that infants in Study 3a looked longer at the cooperation than conflict events because of the preceding Phase 2 events that established the cooperative allegiances. Participants were 25 infants $(11$ male; $M$ age $=15.9$ months, range $=15.6$ 16.4 months). Following the same warm-up as used in Study $3 \mathrm{a}$, children were shown either the conflict or cooperation test events, with no prior Phase 2 events, as in Studies $1 \mathrm{~b}$ and $2 \mathrm{~b}$. Procedures for these test events (including the maximum allowable looking time) were identical to Study 3a. As expected, infants looked equivalently long at the conflict $(M=46.90, S D=$ 13.87) and cooperation $(M=51.76, S D=18.57)$ test events, $t(23)=.75, n s$. Thus, infants did not look longer in the cooperation than conflict test conditions of Study 3a because they generally found cooperation more 
unexpected. Together Studies $3 \mathrm{a}$ and $3 \mathrm{~b}$ confirm that infants can track social allegiances, and use social partnerships to form expectations about behavior based on social interactions alone, even in the absence of salient perceptual cues.

\section{General discussion}

We demonstrated that monitoring the experiences of social partners and using those experiences to form expectations about individual action are functional components of early social cognition. In these violation-of-expectation studies, infants looked longer when an agent cooperated with a target when that agent had previously come into conflict with the target's social partner. They looked longer at these instances of crosspair cooperation only when they had seen a previous instance of cross-pair conflict. They did so when the target agents had never interacted previously (Studies 1a, $2 \mathrm{a}$, and $3 \mathrm{a}$ ) and when nothing differentiated the agents except their individual identities and the patterns of prior interaction of their partners (Study 3a). The critical pattern of results in Studies 1a, 2a, and 3a was obtained even though infants did not find either the cooperation or conflict sequence intrinsically more interesting (as shown in Studies 1b, 2b, and 3b), and when infants' general category knowledge (species differences, Study 1a) and perceptual cues (bandana color, Study 2a) both could and could not (Study 3a) facilitate their expectations on the test trials.

These studies go beyond previous work in infant social cognition, which has focused predominantly on the prediction of action by appeal to individual mental states (Johnson, 2000). This work also goes beyond previous studies of infants' expectations of social interactions (Kuhlmeier et al., 2003; Johnson et al., 2007; Liberman et al., 2014) by showing that infants expect social interactions to shape the subsequent behavior of previously uninvolved social partners. In showing that infants track social allegiances, we establish a critical feature of early social cognition and open a new line of research on the role of social partnerships in social cognition across development (see also Beier et al., 2011; Pietraszewski \& Wynn, 2011). For example, future work could examine the inference processes underlying infants' expectations. There are two separate inference processes that could lead infants to look longer at cross-pair cooperation (following an instance of cross-pair conflict): (1) infants may infer that initial cross-pair conflict will cause later conflict among the social partners, or (2) infants may infer that the initial cross-pair conflict simply provides evidence of ongoing, underlying conflict between the pairs.

Several other critical questions remain regarding the processes that underlie the effects documented here. For example, perhaps infants' behavior in the present studies reflects general expectations that members of pairs interact with each other in characteristic ways that differ from members of other pairs. Such general expectations would enable infants to make a wide range of inferences about behavior (e.g. pair members tend to play one kind of game with each other but not with members of other pairs, or pair members give one kind of greeting to each other but another kind to members of other pairs). On this account, the present findings would reflect general group-oriented mechanisms not specific to reasoning about cooperation or conflict. Alternatively, infants' expectations could be more specific to tracking inter-group cooperation and conflict (e.g. Cosmides, Tooby \& Kurzban, 2003), or events with positive or negative valence (Premack \& Premack, 1997), or for reasoning about helping and harming (Hamlin, 2013). That is, from our data, it is not yet clear how general or specific the mechanisms are that infants use to guide their social partner expectations and inferences. Another critical issue for future work is to determine the extent to which infants interpret the conflict and cooperation interactions presented here as indicative of stable social relationships (such as friendships) that hold predictive power across time and in new situations.

Preschool-age children show some selectivity in their inferences about how agents are influenced by interactions involving their social partners. For example, preschool-age children expect emotional states (e.g. anger) but not physical states (e.g. dizziness) to generalize across social partners (Pietraszewski \& German, 2013). Preschool-age children also expect conflict to generalize across groups (e.g. they expect a fight between individuals from different groups to lead other members of the groups to fight with each other) more than mildly positive behaviors would (e.g. they do not expect sharing between individuals from different groups to lead group partners to share with each other; Chalik \& Rhodes, 2014; Rhodes, 2012). Whether infants' expectations are similarly restricted to certain types of behaviors - or whether such generalizations and specificities develop across the first few years of life - is an important issue for future work.

Perhaps the most intriguing questions concern the developmental origins of and developmental changes in social cognition. The present findings, along with the recent work among preschool-age children described above (Pietraszewski \& German, 2013; Chalik \& 
Rhodes, 2014), suggest some continuity across early human development for this component of social cognition. Yet research in other areas of social cognition has documented important changes from infancy through childhood, as children incorporate early implicit biases into more explicit, articulated theories of human action (Wellman, Cross \& Watson, 2001). Thus, in future work it will be useful to employ similar methods with both younger infants and older children, to identify the full developmental course of this component of social cognition. The present findings also suggest the possibility of an important continuity between this aspect of social cognition in humans with social cognition in nonhuman primates (Cheney \& Seyfarth, 1986, 1999). Such possibilities, and possible future research, become warranted and intriguing based on our demonstration that human infants expect agents' partnerships to shape and predict those agents' actions and interactions.

\section{Acknowledgements}

This research was supported by NIH grant HD022149 to Wellman and NSF grant BCS- 1226942 to Rhodes.

\section{References}

Batson, D. (1998). Altruism and prosocial behavior. In D. Gilbert, S. Fiske \& G. Lindzey (Eds.), The handbook of social psychology (4th edn.) (pp. 282-312). New York: McGrawHill.

Beier, J., Carpenter, M., \& Tomasello, M. (2011, April). Young children's developing understanding of social relationships. In M. Rhodes \& K. Shutts (Chairs), Representations of social relationships in early childhood. Symposium presented at the Biennial Meeting of the Society for Research in Child Development, Montreal, QC.

Chalik, L.A., \& Rhodes, M. (2014). Preschoolers' use of social allegiances to predict behavior. Journal of Cognition and Development, 15, 136-160.

Cheney, D.L., \& Seyfarth, R.M. (1986). The recognition of social alliances by vervet monkeys. Animal Behaviour, 34, 1722-1731.

Cheney, D.L., \& Seyfarth, R.M. (1999). Recognition of other individuals' social relationships by female baboons. Animal Behaviour, 58, 67-75.

Cosmides, L., Tooby, J., \& Kurzban, R. (2003). Perceptions of race. Trends in Cognitive Sciences, 7, 173-178.

Hamlin, J.K. (2013). Moral judgment and action in preverbal infants and toddlers: evidence for an innate moral core. Current Directions in Psychological Science, 22, 186-193.

Hamlin, J.K., \& Wynn, K. (2011). Five- and 9-month-old infants prefer prosocial to antisocial others. Cognitive Development, 26, 30-39.
Hamlin, J.K., Wynn, K., \& Bloom, P. (2007). Social evaluation in preverbal infants. Nature, 450, 557-559.

Hodges, E.V.E., Boivin, M., Vitaro, F., \& Bukowski, W.M. (1999). The power of friendship: protection against an escalating cycle of peer victimization. Developmental Psychology, 35, 94-101.

Johnson, S.C. (2000). The recognition of mentalistic agents in infancy. Trends in Cognitive Sciences, 4, 22-29.

Johnson, S., Dweck, C., \& Chen, F. (2007). Evidence for infants' internal working models of attachment. Psychological Science, 18, 501-502.

Kuhlmeier, V., Wynn, K., \& Bloom, P. (2003). Attribution of dispositional states by 12-month-olds. Psychological Science, 14, 402-408.

Liberman, Z., Kinzler, K.D., \& Woodward, A.L. (2014). Friends or foes: infants use shared evaluations to infer others' social relationships. Journal of Experimental Psychology: General, 143, 966-971.

Onishi, K., \& Baillargeon, R. (2005). Do 15-month-old infants understand false beliefs? Science, 308, 255-258.

Papachristos, A.V. (2009). Murder by structure: dominance relations and the social structure of gang homicide. American Journal of Sociology, 115, 74-128.

Pietraszewski, D., \& German, T. (2013). Coalitional psychology on the playground: reasoning about indirect social consequences in preschoolers and adults. Cognition, 126 (3), 352-363.

Pietraszewski, D., \& Wynn, K. (2011, April). Evidence of a core inference of group psychology in preverbal infants: young children's developing understanding of social relationships. In M. Rhodes \& K. Shutts (Chairs), Representations of social relationships in early childhood. Symposium presented at the Biennial Meeting of the Society for Research in Child Development, Montreal, QC.

Platten, L., Hernik, M., Fonagy, P., \& Fearon, R.P. (2010). Knowing who likes who: the early developmental bias of coalition understanding. European Journal of Social Psychology, 40, 569-580.

Premack, D., \& Premack, A.J. (1997). Infants attribute value \pm to the goal-directed actions of self-propelled objects. Journal of Cognitive Neuroscience, 9, 848-856.

Repacholi, B., \& Gopnik, A. (1997). Early reasoning about desires: evidence from 14- and 18-month-olds. Developmental Psychology, 33, 12-21.

Rhodes, M. (2012). Naïve theories of social groups. Child Development, 83, 1900-1916.

Wellman, H.M., Cross, D., \& Watson, J. (2001). Meta-analysis of theory-of-mind development: the truth about false belief. Child Development, 72, 655-684.

Woodward, A. (1998). Infants selectively encode the goal object of an actor's reach. Cognition, 69, 1-34.

Received: 9 November 2012

Accepted: 4 September 2014 